This document is the Accepted Manuscript version of a Published Work that appeared in final form in British Journal of Midwifery, copyright (C) MA Healthcare, after peer review and technical editing by the publisher. To access the final edited and published work see https://doi.org/10.12968/bjom.2017.25.9.579 


\section{Tongue-tie and breastfeeding: Identifying problems in the diagnostic and treatment journey}

Rachel Wakelin, general practitioner ${ }^{1}$

Andrea Stöckl, lecturer in the sociology of medicine ${ }^{2}$

Amanda Howe, clinical professor in primary care ${ }^{2}$

Robert Fleetcroft, honorary senior fellow in primary care ${ }^{2}$

1) St Marys Surgery, Ely,

2) Norwich Medical School, Norfolk NR4 7TJ 


\section{Abstract}

\section{Background}

Tongue-tie is a common condition which often adversely affects breastfeeding. There is research that suggests that frenulotomy can improve breastfeeding but there is evidence of lack of professional knowledge on tongue-tie

\section{Aim and Methods}

We undertook a qualitative interview study with GPs, midwives and health visitors and 9 mothers to explore facilitators and barriers to receiving a diagnosis of and treatment for tongue-tie.

Results

Mothers told a common story of having to push for support, diagnostic and treatment delay, and ongoing distress; which threatens a mother's ability to establish breastfeeding. Mothers described vulnerabilities in the neonatal period, and variation in professional knowledge about tongue-tie.

\section{Conclusion}

Variable professional knowledge, conflicting advice, and delayed diagnosis can lead to a difficult patient pathway. Assessment for tongue-tie should be considered when approaching infants with feeding difficulties and frenulotomy should be considered and services available where breastfeeding support has not helped. 


\section{Background}

Tongue-tie (ankyloglossia) is a common condition with a prevalence between 0.2 and $10.7 \%$. (Francis et al., 2015, Power and Murphy, 2015, Segal et al., 2007) It is an embryological remnant of tissue between the undersurface of the tongue and the floor of the mouth that can restrict tongue movement. This is an important condition for primary care because it can cause breastfeeding difficulty for the mother and infant; including nipple pain, difficult attachment and therefore increased bottle feeding rates. (Edmunds et al., 2011, Segal et al., 2007, Suter and Bornstein, 2009) Mothers have described an "anticipatory dread" towards breastfeeding an infant with

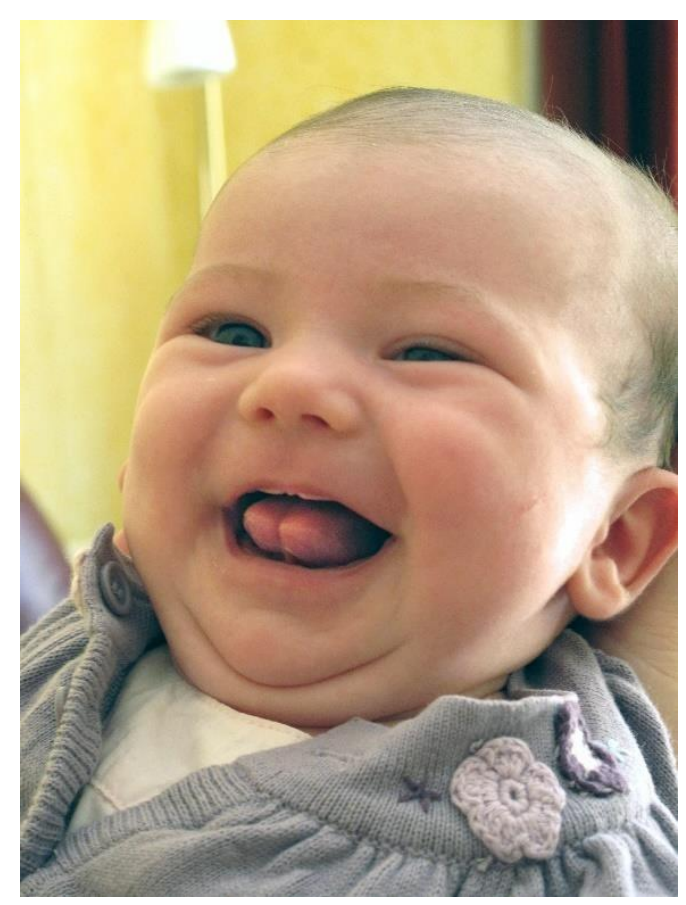

Figure 1. Tongue-tie can be obvious such as with visualisation of a restricting frenulum or a heart-shaped tongue but appearance can vary and some tongue-ties are hard to visualise. tongue-tie. (Edmunds et al., 2013) Tongue-tie can be obvious, such as with a restricting frenulum or heart-shaped tongue, but others may only be diagnosed after assessment of: breastfeeding difficulties, positioning, attachment, tongue appearance and function, by practitioners with the appropriate expertise. (Hill and Johnson, 2007) Other causes of difficult feeding such colic, reflux, poor attachment, positioning, and inverted nipples would need to be considered. (Amir, 2014) Assessment for tongue-tie is not part of routine UK neonatal examination and is usually identified by GPs, midwives or health visitors in the context of emerging breastfeeding problems. Tongue-tie, if it is symptomatic, can be treated with a frenulotomy, which involves snipping the tongue with sharp, round scissors. Bleeding is stopped by allowing the baby to feed, which compresses the wound. (Edmunds et al., 2011) In 2005, UK guidelines from the National Institute of Clinical Excellence (NICE) conclude that limited evidence suggests that division of tongue-tie is both safe and can improve breastfeeding where conservative measures such as frenulum massage and breastfeeding support have failed. (Interventional Procedures Advisory Committee, 2005) Although there is no published research to recommend frenulum massage.

Eight systematic reviews (Finigan and Long, 2013, Ito, 2014, Power and Murphy, 2015, Segal et al., 2007, Webb et al., 2013, Suter and Bornstein, 2009, Francis et al., 2015, Edmunds et al., 2011) have 
been undertaken in this area which highlight the clinical uncertainty surrounding management of tongue-tie. Five reviews reported that compared to routine breastfeeding support, frenulotomy can improve breastfeeding difficulties. (Finigan and Long, 2013, Ito, 2014, Power and Murphy, 2015, Webb et al., 2013) Finigan and Long conclude that there is evidence for improvement in more than $50 \%$ of the cases and Edmunds et al. go on to say that it would be unethical not to provide treatment where benefit has been shown. (Edmunds et al., 2011, Finigan and Long, 2013) Two reviews draw more tentative conclusions that there is a small body of evidence to suggest that frenulotomy can offer improvement and confidence in that evidence is low. (Francis et al., 2015, Segal et al., 2007) Suter and

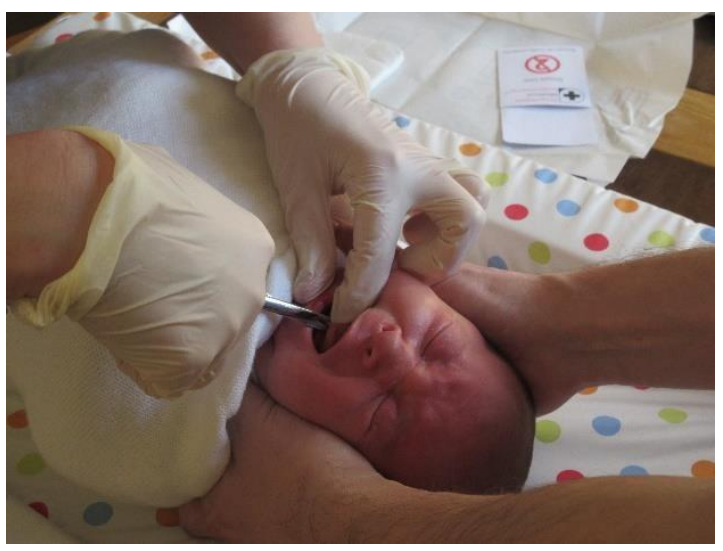

Figure 2. Tongue-tie, if it is symptomatic, can be treated with a frenulotomy, which involves snipping under the tongue with sharp, round scissors.
Bornstein felt that while breastfeeding problems could be associated with tongue-tie in $25-80 \%$ of cases, due to inability to make comparisons between studies, they could not draw conclusions to resolve controversy in this area. (2009) Ambivalent conclusions were universally due to: the heterogeneous nature of the research, use of diverse diagnostic and outcome measures; inadequate comparison of conservative management and lack of robust study design.

Although not overwhelming, there is a growing body of research that suggests that some infants with tongue-tie need additional breastfeeding support with or without frenulotomy, but there is evidence of a reluctance to refer for or lack of knowledge about frenulotomy. This is understandable given the limited evidence, but this may impact on the quality of care for these infants. Two studies have identified diverse and contrasting views between paediatricians, otolaryngologists and surgeons for frenulotomy (Messner and Lalakea, 2000, Brinkmann et al., 2004) and a third study described mothers encountering professionals who rarely identified tongue-tie as a problem or gave conflicting advice. (Edmunds et al., 2013) In addition referral routes for frenulotomy vary around the UK and can cause confusion for parents and referrers; procedures are performed by otolaryngologists, maxillofacial surgeons, paediatric surgeons, midwives, health visitors or lactation consultants. Treatment is also widely available through private tongue-tie practitioners. It is unclear how soon is ideal to perform a frenulotomy to support the establishment of breastfeeding, however there is evidence of a substantial wait for treatment within the 
UK: a freedom of information request to NHS trusts found the longest wait for frenotomy, after diagnosis, was 84 days and the average 21.6 days. (Boffey, 2014)

\section{Objectives}

Overall, the literature provides evidence that tongue-tie can cause breastfeeding problems that may be improved by frenulotomy, but that this is often not reflected in practice and can lead to diagnostic and treatment delay. This study aimed to explore the experience of parents and primary care professionals, exploring knowledge and opinions on tongue-tie in breastfeeding and experience of the diagnostic and treatment pathway within the UK. With the hope of understanding how we can improve treatment pathways for infants with tongue-tie even in the context of clinical uncertainty.

\section{Methods}

A qualitative case study approach was used with 23 participants; this sample size is in keeping with wider qualitative research practice as it was felt data saturation had been reached and further interviews would not elicit further information. (Mason, 2010) We undertook semi-structured telephone interviews with five general practitioners (GPs), four midwives and five health visitors and a focus group with nine mothers. The women were recruited from one of three local 'free to attend' breastfeeding clinics and National Health Service professionals were recruited from 25 training general practices in Cambridgeshire, UK where the lead research was based. All procedures were performed in compliance with University of East Anglia guidelines and approval was received from the Faculty of Medicine and Health Sciences Research Ethics Committee (reference 20142015-63).

As large scale random sampling to achieve generalisability is not possible with such intensive research methods purposive sampling was undertaken to attempt to gain transferability to the wider population. (Silverman, 2005) The inclusion criteria were women with breastfed infants aged seven months or younger; we aimed to achieve a mixed sample and participants were stratified to reflect rural and urban backgrounds and different pathways to clinic. Women who had breastfed older children with tongue-tie were not included in the study, nor mothers of babies born prematurely or with significant health problems, or who were not confident to communicate in English. A mixed gender sample was selected of three female and two male GPs and all female midwife and health visitor participants. Professionals 
were selected from a mixture of urban and rural settings with a range of years practicing. No professionals with specific training in tongue-tie were included in the study.

Data were collected over a four-month period from June to October 2015. The focus group was conducted over 60 minutes with a facilitator and observer. A topic guide was used covering four areas: knowledge about tongue-tie; diagnosis; clinical assessment of tongue-tie and referral practices. Practitioners were interviewed by telephone; questions explored experience of tongue-tie and breastfeeding, thoughts on the impact of tongue-tie and frenulotomy, their examination and referral practices, their knowledge and opinions. The interviews were recorded digitally and transcribed verbatim. Data were analysed in Microsoft Word and Excel 2013 using thematic analysis based on a grounded theory approach. (Charmaz, 2006) Codes were assigned to each line and then grouped into categories and links made between codes and categories. A sample of initial codes were derived separately by two of the authors to avoid bias and discrepancies in approach to coding were resolved. Pseudonyms were used to preserve anonymity.

\section{Results}

Nine mothers participated; for eight, this was their first child. All the women in this study had wanted to breastfeed but had universally experienced difficulties in feeding. They described: vulnerabilities, by recounting feeling "out of it" post-partum; receiving sometimes conflicting advice on tongue-tie and breastfeeding; and feeling "desperate" to have a frenulotomy. Health visitor, Kacy, described some of the mothers she has seen having problems with tongue-tie:

...they are pretty desperate because of their wish to breastfeed, the pain and also desperately trying to do what they feel is the right thing for their baby and them just not managing. And the babies tend to be irritable and grisly and you know not having a good time of it either.

For the infants of the mother participants the average age of tongue-tie diagnosis was 18.4 days (range $0-70$ ), the average age of treatment was 25.3 days (range 5-70); All infants received a frenulotomy and six of the nine were done privately. Mothers discussed at length how they felt that they were "lucky" to see the right person and examples were given of healthcare professionals who seemed to have, "no idea" about tongue-tie or where tongue-tie diagnoses were missed. Mothers described consulting 
"multiple" clinics and professionals for diagnosis. For some, the diagnoses were made at breastfeeding clinics, then refuted by physicians and then later reconfirmed. Wendy talks of her distress when seeking advice about tongue-tie,

I went to the GP, they said they don't know anything about tongue-tie. And it's the horrible thing that no one, they tell you, but you don't know where to go. You're so alone in there.

In contrast many participant practitioners demonstrated a breadth of knowledge about tongue-tie and discussed the necessity for examining the tongue in certain situations and five mothers said that they had received their baby's tongue-tie diagnosis within three days of birth. Mothers also talked about the helpful advice they had received about attachment and positioning.

Mothers discussed their surprise that many practitioners did not examine their babies' mouths, frustrated at it not being part of the routine check and frustrated with healthcare practitioners' lack of skills in this area and describe being "lucky" to see the right practitioner. Esther compares her experience when a practitioner made the diagnosis to her previous encounter with professionals,

No one else, I don't think, listened to the whole story. So they won't latch, she won't

latch... But also, no one listened to the "and they're feeding for hours and there's this and there's that" They just wanted to look at a little bit of the picture.

Aside from knowledge, attitudes towards frenulotomy amongst practitioners also varied: two GPs felt they didn't have enough information to comment on this, many practitioners emphasised that the need for it just "depends" on the clinical picture, and several practitioners emphasised how important they felt frenulotomy can be. In contrast, there were some more negative views about breastfeeding and the diagnosis of tongue-tie with some professionals dismissing its existence or significance, others referring to it as a "hobby horse" of the midwives or a "trend term". Mothers described feeling dismissed with both negative remarks and body language as well as receiving unhelpful or conflicting advice; being told to just "toughen up", that they were "doing something wrong" or that they had a "lazy baby". Louise discusses an encounter with a paediatrician after her tongue-tie diagnosis was refuted, 
She was like, if it's really bad, you can put him on formula. And we both looked at each other, but that's not an option. She stood on the side, leaning with her arms crossed and just didn't answer. So we just got up and walked out. I was so upset.

Professional knowledge on breastfeeding was in the form of embodied knowledge, which is subjective knowledge gained through personal experience of breastfeeding a baby; vicarious or cultural knowledge, through observing a breastfeeding experience; practice-based knowledge gained through clinical experience; and formal knowledge from structured learning opportunities, literature and web based sources. Amongst practitioners there was varied knowledge of national guidelines and all expressed some lack of confidence regarding their knowledge base or ability to diagnose tongue-tie. While the health visitors and midwives had received professional training on tongue-tie in recent years, it was something that all professionals reported as mostly absent from their previous training. While clinical experience seemed to be the key source of knowledge, several healthcare professionals had personal experience of tongue-tie which clearly influenced their attitudes. This was seen in comments regarding themselves, "based on my own personal experience...", or in observations of others; Midwife Shellie discussed a paediatrician who she felt "knows the evidence" but "because his wife successfully breastfed their baby with a tongue-tie he sort of won't address it." Issues of multi-disciplinary dialogue were also raised with evidence of limited discussion about tongue-tie between GPs and midwives or health visitors. It was clear that there were differing epistemological approaches to understanding tongue-tie; with disciplines finding greater value in theoretical, embodied or vicarious knowledge. There was also a lesser emphasis on promotion of breastfeeding from GPs; two GPs discussed that they felt that as GPs they needed to put on less pressure to breastfeed and one midwife, Shellie comments,

You know I still think there is a feeling among some people, oh if you don't breastfeed it is okay, you just formula feed don't you...It is deemed to be an equal alternative.

Most participants raised concerns that frenulotomies were often performed within the private sector; with many professionals expressing unease or confusion about how to share information about private practice with mothers as this was not their usual practice. Others raised questions about a financial conflict of interest, follow-up may be inadequate or costly, or that this took advantage of vulnerable mothers who are in a "desperate" situation and would "pay anything". In contrast, professionals and 
mothers also talked about tongue-tie practitioners who provided: a wealth of experience, usually as exmidwives or health visitors; as well as prompt assessment; adequate time to fully listen and examine and good follow-up care. Mothers discussed with some passion that they felt lucky that they could afford to go privately but felt strongly that this was "far too much money" and unfair for those less fortunate; practitioners also discussed this inequity.

\section{Conclusion}

\section{Summary}

Our account has painted a picture of vulnerable patients: experiencing breastfeeding difficulties while meeting the challenges of a new baby; in a system that has resource limitations, organizational difficulties and a private-NHS interface. This is in the context of variable professional knowledge and opinions, conflicting advice and diagnostic conclusions. This led to a difficult patient pathway; mothers told a common story of having to push for support, attending multiple appointments, diagnostic and treatment delay and ongoing distress. They described feeling "lucky" to receive a diagnosis, sometimes lost in the system, frustrated and desperate.

\section{Strengths and limitations}

The research had a strong insider perspective with the research lead being both a GP and mother and having had experience of breastfeeding an infant with tongue-tie, which enhanced the research process. Interview technique was designed to be facilitatory rather than conversational, established data analysis techniques and triangulation of data from a range of participants were used to attempt to mitigate again bias from the insider perspective. The sample size is small and from one geographic area but included a range of practitioners and mothers had a variety of referral routes to the breastfeeding clinic that they were recruited from. Eight of our patients were from Caucasian background and only one did not hold a higher degree however this fits with known breastfeeding patterns where a higher proportion of breastfeeding mothers in the UK are Caucasian and from professional and intermediate occupations. (McAndrew et al., 2012) Six of our patients had their frenulotomy undertaken privately and a shorter wait to treatment, and as the prevalence of private frenulotomies within the UK is undocumented it is unclear as to whether this is very different from wider treatment practice. In addition, due to recruitment problems many of the mothers were first time mothers 
and thus their problems may be amplified. However, the majority of study participants are healthcare professionals and thus bring a breadth of experience that offers some balance to the selection bias seen within the mother participants.

\section{Comparison with existing literature}

Although there are some design limitations many of our findings are supported by previous research. The experience of mothers in our study is akin to the difficult journey illustrated by Edmunds et al. (2013) Although in our group there was a shorter wait between diagnosis and treatment of 6.9 days (range 0 24) compared to a UK average of 21.6 identified by Boffey. (2014) Variability in knowledge regarding tongue-tie has also been previously demonstrated (Messner and Lalakea, 2000) as has lack of knowledge and confidence towards breastfeeding in general. (Smale et al., 2006) Smale et al. documents the lack of prioritisation of breastfeeding education, (2006) which is mirrored by lack of tongue-tie in training in this study. The inconsistent approaches we have discussed towards tongue-tie have also been seen by Dykes in wider breastfeeding care as well as differing paradigmatic stances between professionals. (Dykes, 2006) It was clear in this study that different disciplines had different epistemological stances; stemming from the extent of their different sources of breastfeeding knowledge; including theoretical, embodied and vicarious; but also due to the impact that different types of knowledge had on their opinion.

\section{Implications for research and practice}

We support the need for further investigation into the impact of tongue-tie and effectiveness of frenulotomy including studies with newly developed tools such as the Bristol Tongue Assessment Tool. (Ingram et al., 2015)

Educationally, we would recommend an increased emphasis on breastfeeding training, including discussion around tongue-tie and the referral routes for management; this would include within undergraduate and pre-registration training and as part of continuing professional development. Undergraduate training could be through the Baby Friendly Initiative (2014) University Standards Programme which provides learning outcomes to be met by midwives and health visitors to provide basic breastfeeding knowledge. Importantly this includes drawing on knowledge and understanding of wider influences that affect breastfeeding such as the social, cultural and political context which is 
essential to provide holistic support of breastfeeding. In addition to this, training also needs to develop skills to learn from formal, embodied and vicarious knowledge especially within the context of clinical uncertainty. Key practitioners such as midwives, health visitors and lactation consultants should also be adequately trained to be able to be able to diagnose tongue-tie.

Practice based issues have also been partly described in wider breastfeeding care. In existing pathways, there may be potential to improve diagnosis of tongue-tie. There is not enough evidence on the benefits and potential harms from frenulotomy and other treatments for tongue-tie to support screening for this condition. However, guidelines on tongue-tie, such as those from NICE, could be extended from just suggesting that it is a safe procedure to encompass guidance on education, consideration of tongue-tie diagnosis when evaluating feeding problems, and universal provision of the frenulotomy when clinically indicated. The role of clinicians, without expertise in tongue-tie diagnosis, would be to refer mothers on for further evaluation by someone with skills in breastfeeding and tonguetie assessment. Issues of discordance of approach and inadequate dialogue between the multiprofessional team need to be considered in education and clinical pathway design.

Finally, we have raised ethical and professional issues in a national health service system with vulnerable parents making desperate decisions about whether to pay for private treatment. In a public health system, such as the NHS, treatment pathways need to be clear, timely and accessible.

\section{Key phrases}

1. Tongue-tie can detrimentally impact breastfeeding and frenulotomy affords improvement but needs much further research.

2. The current lack of professional knowledge calls for increased breastfeeding and tongue tietraining for all healthcare professionals involved in postnatal care.

3. The current pathway from tongue-tie diagnosis to treatment is problematic; this could be improved through more clarity in the role of GPs, midwives and health visitors in problem identification, signposting, diagnosis and referral. More clinicians with breastfeeding expertise should be equipped to feel confident to diagnose tongue-tie.

4. National guidelines on tongue-tie, such as those from NICE, could be extended to encompass: guidance on education, a prompt to consider tongue-tie diagnosis when evaluating feeding 
problems, and direction to provide universal, timely and accessible frenulotomy when it is clinically indicated.

\section{References}

Amir, L. H. (2014) 'Managing common breastfeeding problems in the community', Bmj, 348, pp. g2954.

Baby Friendly Initiative 2014. Implementing the UNICEF UK Baby Friendly standards in universities:

Learning outcomes and topic areas. London.

Boffey, D. (2014) Concern over delays to treatment of babies suffering from tongue-tie: theguardian.com. Available at: http://www.theguardian.com/society/2014/jul/12/tonguetie-babies-delays-treatment-nhs-breastfeeding (Accessed: 5th January 2015.

Brinkmann, S., Reilly, S. and Meara, J. G. (2004) 'Management of tongue-tie in children: a survey of paediatric surgeons in Australia', Journal Of Paediatrics And Child Health, 40(11), pp. 600-605.

Charmaz, K. (2006) Constructing Grounded Theory: A Practical Guide through Qualitative Analysis. SAGE Publications.

Dykes, F. (2006) 'The education of health practitioners supporting breastfeeding women: time for critical reflection', Maternal \&amp; Child Nutrition, 2(4), pp. 204-216.

Edmunds, J., Miles, S. C. and Fulbrook, P. (2011) 'Tongue-tie and breastfeeding: a review of the literature', Breastfeed Rev, 19(1), pp. 19-26.

Edmunds, J. E., Fulbrook, P. and Miles, S. (2013) 'Understanding the experiences of mothers who are breastfeeding an infant with tongue-tie: a phenomenological study', J Hum Lact, 29(2), pp. 190-5.

Finigan, V. and Long, A. (2013) 'The effectiveness of frenulotomy on infant-feeding outcomes: A systematic review', Evidence Based Midwifery, 11(2), pp. 5.

Francis, D. O., Krishnaswami, S. and McPheeters, M. (2015) 'Treatment of ankyloglossia and breastfeeding outcomes: a systematic review', Pediatrics, 135(6), pp. e1458-e1466.

Hill, P. D. and Johnson, T. S. (2007) 'Assessment of breastfeeding and infant growth', J Midwifery Womens Health, 52(6), pp. 571-8.

Ingram, J., Johnson, D., Copeland, M., Churchill, C. and Taylor, H. (2015) 'The development of a new breast feeding assessment tool and the relationship with breast feeding self-efficacy', Midwifery, 31(1), pp. 132-7.

Interventional Procedures Advisory Committee 2005. Interventional procedures overview of division of ankyloglossia (tongue-tie) in babies with difficulty breastfeeding. London: National Institute of Clinical Excellence.

Ito, Y. (2014) 'Does frenotomy improve breast-feeding difficulties in infants with ankyloglossia?', Pediatrics International: Official Journal Of The Japan Pediatric Society, 56(4), pp. 497-505.

Mason, M. (2010) 'Sample size and saturation in PhD studies using qualitative interviews', Forum: Qualitative Social Research/Forum Qualitative S ozialforschung, 11. 
McAndrew, F., Thompson, J., Fellows, L., Large, A., Speed, M. and Renfrew, M. J. (2012) Infant Feeding Survey $2010 \quad$ Consolidated Report. Available at: http://www.hscic.gov.uk/catalogue/PUB08694/Infant-Feeding-Survey-2010-ConsolidatedReport.pdf.

Messner, A. H. and Lalakea, L. (2000) 'Ankyloglossia: controversies in management', International Journal of Pediatric Otorhinolaryngology, 54(Issues 2-3), pp. 123-131.

Power, R. F. and Murphy, J. F. (2015) 'Tongue-tie and frenotomy in infants with breastfeeding difficulties: achieving a balance', Archives of Disease in Childhood, 100(5), pp. 489-494 6p.

Renfrew, M. J. (2006) 'Time to get serious about educating health professionals', Maternal \& Child Nutrition, 2(4), pp. 193-195.

Segal, L. M., Stephenson, R., Dawes, M. and Feldman, P. (2007) 'Prevalence, diagnosis, and treatment of ankyloglossia: methodologic review', Canadian Family Physician, 53, pp. 1027-1033 7p.

Silverman, D. (2005) Doing Qualitative Research: A Practical Handbook. SAGE Publications.

Smale, M., Renfrew, M. J., Marshall, J. L. and Spiby, H. (2006) 'Turning policy into practice: more difficult than it seems. The case of breastfeeding education', Maternal \&amp; Child Nutrition, 2(2), pp. 103-113.

Suter, V. G. and Bornstein, M. M. (2009) 'Ankyloglossia: facts and myths in diagnosis and treatment', J Periodontol, 80(8), pp. 1204-19.

Webb, A. N., Hao, W. and Hong, P. (2013) 'The effect of tongue-tie division on breastfeeding and speech articulation: a systematic review', Int J Pediatr Otorhinolaryngol, 77(5), pp. 635-46. 